\title{
Article
}

\section{Genetic and Environmental Influences on Vigorous Exercise in South Korean Adolescent and Young Adult Twins}

\author{
Yoon-Mi Hur \\ General College of Education, Kookmin University, Seoul, South Korea
}

\begin{abstract}
Twin studies of physical exercise for Asian twins are sparse. This study aimed to examine genetic and environmental influences on frequency of vigorous exercise (FVE) in South Korean twins, with a special emphasis on sex effects. Telephone interviews on FVE were administered to 1757 twins (mean age $=19.05$ years, $S D=3.01$ years). Tetrachoric correlations were significantly different between monozygotic $(\mathrm{MZ})$ and dizygotic (DZ) twins in males (.40 vs. .12), but they were similar in females (.44 vs. .45), suggesting the importance of genetic factors in FVE in males and that of common environmental factors in females. A scalar sex-limitation model incorporating age as a modifier was applied to data. The results revealed that genetic, common and individual environmental influences did not vary significantly with age, but differed across two sexes, confirming twin correlational analyses. In the best-fitting model, additive genetic and individual environmental influences on FVE were, respectively, .35 (95\% CI $[.26, .39])$ and .65 (95\% CI $[.61, .74])$ in males, and common and individual environmental influences were, respectively, .45 (95\% CI $[.35, .53])$ and $.55(95 \%$ CI $[.47, .65])$ in females. These results contrasted starkly with recent findings from a large sample of Chinese adult twins (age $>18$ years), in which most variance ( $\geq 95 \%$ ) of vigorous physical activity was attributable to common environmental influences in both sexes. Replications in other Asian samples are clearly needed.
\end{abstract}

Keywords: vigorous exercise; twin study; heritability; common environmental influences; Asians

(Received 18 January 2021; accepted 3 February 2021; First Published online 19 March 2021)

The beneficial effects of physical exercise on health and quality of life are well documented. For example, a higher level of physical exercise is associated with a lower risk of developing obesity (Hills et al., 2011), cardiovascular disease (Wannamethee \& Shaper, 2001), type 2 diabetes (Aune et al., 2015), mental health problems (Grasdalsmoen et al., 2020) and various cancers (Brown et al., 2012). Additionally, physical exercise has positive effects on psychological wellbeing (Bartels et al., 2012), academic performance in adolescents and young adults (Aaltonen et al., 2020) and cognition in the elderly (Iso-Markku et al., 2018). Despite these well-known benefits of physical exercise, during the past decades, the prevalence of insufficient physical activity has increased worldwide (Guthold et al., 2018). Thus, it is important to investigate determinants of individual differences in exercise behavior in various populations for development of optimal exercise intervention strategies and policy guidelines for global health promotion.

Previous twin studies have shown that genetic factors influence exercise behavior, even though the estimates of genetic influences have varied substantially by measure, age and sex (de Geus et al., 2014). In Dutch twin studies of physical exercise (de Moor et al., 2011; van der Aa et al., 2010; Vink et al., 2011), genetic influences were small in childhood $(20 \%-40 \%)$ but increased from childhood

Author for correspondence: Yoon-Mi Hur, Email: ymhur@kookmin.ac.kr

Cite this article: Hur Y-M. (2021) Genetic and Environmental Influences on Vigorous Exercise in South Korean Adolescent and Young Adult Twins. Twin Research and Human Genetics 24: 116-122, https://doi.org/10.1017/thg.2021.6 to adolescence $(38 \%-85 \%)$ and then decreased in adulthood (about 40\%). In contrast, common environmental influences were substantial in childhood $(50 \%-75 \%)$ but mostly nonsignificant afterward. Individual environmental influences were generally small during childhood and adolescence $(<28 \%)$ but became increasingly large (about $60 \%$ ) with age in adulthood. In a Finnish longitudinal twin study of physical exercise, Aaltonen et al. (2013) found that in both sexes the heritability of leisure-time physical activity remained significant (43\% to $52 \%$ ) during adolescence (16-18 years) but reduced to approximately $30 \%$ in young adulthood (about 24 years). In contrast, common environmental influences increased from adolescence (18\% to $26 \%$ ) to young adulthood (43\% to $49 \%$ ). Individual environmental influences remained relatively stable across adolescence and young adulthood, ranging from $20 \%$ to $30 \%$ in both sexes. The GenomeEUtwin project (Stubbe et al., 2006; Vink et al., 2011), which pooled adult twin data from seven European countries (Australia, Denmark, Finland, the Netherlands, Norwey, Sweden and the UK), revealed that genetic influences on physical activity ranged from $48 \%$ to $71 \%$ across the countries (Stubbe et al., 2006) and that heritability reduced from about $70 \%$ to $40 \%$ with increasing age from 19 to 50 years, whereas individual environmental influences increased from about $30 \%$ to $60 \%$ in four of the seven countries. With a few exceptions, common environmental influences were absent across the adulthood (Vink et al., 2011).

Sex differences in genetic and environmental influences on physical exercise were significant in some studies (e.g. Carlsson

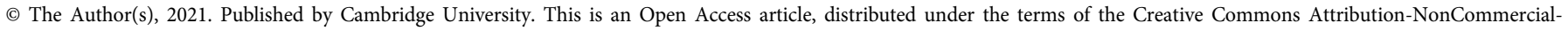

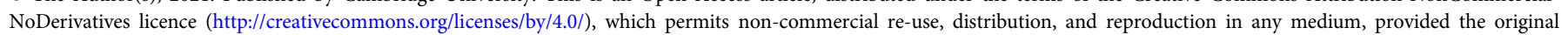
work is unaltered and is properly cited. The written permission of Cambridge University Press must be obtained for commercial re-use or in order to create a derivative work. 
et al., 2006; Maia et al., 2002) but not in others (e.g. van der Aa et al., 2010; Vink et al., 2011). Whenever significant differences were found, males tended to have higher genetic and lower common environmental influences than did females. For example, van der Aa et al. (2010) found that $85 \%$ and $38 \%$ of variability in leisure time physical activity at ages 13-14 years were explained by genetic factors for boys and girls, respectively, only in girls were $46 \%$ accounted for by common environmental influences. In another example, a Portuguese twin sample aged from 12 to 25 years showed that genetic influences were higher $(63 \%$ vs. $32 \%)$, and common environmental influences were lower $(0 \%$ vs. $38 \%$ ) in males than in females, whereas individual environmental influences were roughly similar (37\% vs. $30 \%)$ in two sexes (Maia et al., 2002).

Genetic and environmental influences on physical activity in Asians remain poorly understood because most twin studies of exercise to date have investigated American and European samples. In a large sample of Chinese adult same-sex twins (age $>18$ years, $N=19,308$ ), Wang et al. (2016) found that individual difference in vigorous physical activity defined as 150 minutes of moderate or high-intensity physical activity per week was almost completely explained by common environmental influences in both sexes ( $95 \%$ in males, $98 \%$ in females) and that genetic influences were inconsequential ( $<3 \%$ in both sexes). These results sharply contrasted those found in most Western twin samples. Discrepancies in findings cannot be attributed to measurement differences because, like many Western twin studies, the Wang et al. study employed the questionnaire method. Differences in race/ethnic differences in physical fitness and exercise abilities might have contributed to the discrepancies in results. Thus, it is important to further explore genetic and environmental influences on physical activity in Asian samples which have genetic backgrounds similar to Chinese twins. If Asians are vastly different from Westerners in genetic and environmental etiologies of physical activity, then ethnically sensitive exercise intervention strategies and guidelines might be needed for effective health promotion. Thus, the present study investigated genetic and environmental influences on frequency of vigorous exercise (FVE) in South Korean adolescent and young adult twins, with a special emphasis on sex effects.

\section{Methods}

\section{Sample}

The total sample included 1757 adolescent and young adult twins (mean age $=19.05$ years, $S D=3.01$ years) identified from the South Korean Twin Registry (SKTR; Hur et al., 2019). The SKTR is a nationwide volunteer registry of South Korean preschooler to young adult twins and their families. Adolescent twins in the SKTR were collected from schools throughout South Korea, whereas young adult twins were from Facebook, twin clubs and colleges throughout South Korea. Various psychological assessments have been administered via telephone and mail surveys to twins registered with the SKTR. Zygosity of the twins in the SKTR was assessed through three items asking twins' physical similarity and frequency of confusion by family members, close friends and strangers (Ooki et al., 1993).

The total sample consisted of 368 monozygotic male (MZM) twins (155 complete pairs and 58 twin individuals), 173 dizygotic male (DZM) twins (67 complete pairs and 39 twin individuals), 682 monozygotic female (MZF) twins (312 complete pairs and 58 twin individuals), 274 dizygotic female (DZF) twins
(117 complete pairs and 40 twin individuals) and 260 oppositesex dizygotic (OSDZ) twins (119 complete pairs and 22 twin individuals). Larger numbers of $\mathrm{MZ}$ than $\mathrm{DZ}$ twins likely reflected the twin birth rates in the South Korean population for the birth cohorts in the present study (Hur \& Kwon, 2005; Hur \& Song, 2009).

\section{Measures}

During the SKTR telephone interview, twins were asked how often they engaged in vigorous physical exercise. Interviewers explained 'vigorous exercise' as that intense enough to cause rapid breathing and/or sweating. There were four response categories ( $0=$ no exercise, $1=1-2$ times per week, $2=3-4$ times per week $3=5$ times or more per week). Interviewers also asked twins who chose the response category of 1 or higher if they exercised more or less than 30 minutes each time, and $83 \%$ reported that they exercised a minimum of 30 minutes or more. The remaining $17 \%$ reported that they exercised less than 30 minutes each time.

\section{Statistical Analysis}

Data analyses were conducted in the following steps. First, descriptive analyses examined relationships of FVE with sex, age and zygosity. As twins are not an independent sample, phenotypic analyses were performed using one randomly chosen member of each twin pair in descriptive analyses. Second, as a preliminary step examining genetic, common environmental and individual environmental influences on FVE, tetrachoric correlations with 95\% confidence intervals (CI) for each type of twins were computed. Finally, a scalar sex-limitation model extended to include age as a moderator was fitted to data to estimate genetic and environmental contributions to individual differences in FVE in males and females and to investigate whether these contributions change with age during adolescence and young adulthood.

Because FVE is an ordinal variable, a liability threshold model was used to analyze twin data. The basic assumption underlying the liability threshold model is that a latent continuous liability underlies the distribution of the ordinal variable. The liability is assumed to have standard normal distribution with a mean of zero and standard deviation of 1.0. To model the four categories of vigorous exercise in the present study, three thresholds were required. The thresholds, expressed in $z$ values, are defined by the prevalence of the four distinct categories of vigorous exercise frequencies. Using the raw data, $\mathrm{MZ}$ and $\mathrm{DZ}$ twin tetrachoric correlations were computed. As MZ twins share $100 \%$ of their segregating genes, but DZ twins, on average, share only $50 \%$, higher MZ than DZ twin correlation indicates the presence of additive genetic effects (i.e. the sum of the average effect of all alleles that influence a trait). On the other hand, DZ correlation higher than half the MZ correlation indicates the presence of common environmental effects (i.e. those environmental factors shared between the two members of a twin pair).

A path diagram of the scalar sex-limitation model incorporating the age moderator is shown in Figure 1. In the model, the total variance of FVE was divided into additive genetic (A), common environmental (C) and individual environmental effects (E; i.e. those environmental factors unique to each member of a twin pair and measurement error). Correlations for additive genetic effects for $\mathrm{MZ}$ and DZ twins were fixed at 1.0 and .5, respectively, for each sex, whereas common environmental effects were fixed at 1.0 for both $\mathrm{MZ}$ and DZ twins. Individual environmental effects were uncorrelated between the two members of a twin pair, regardless 


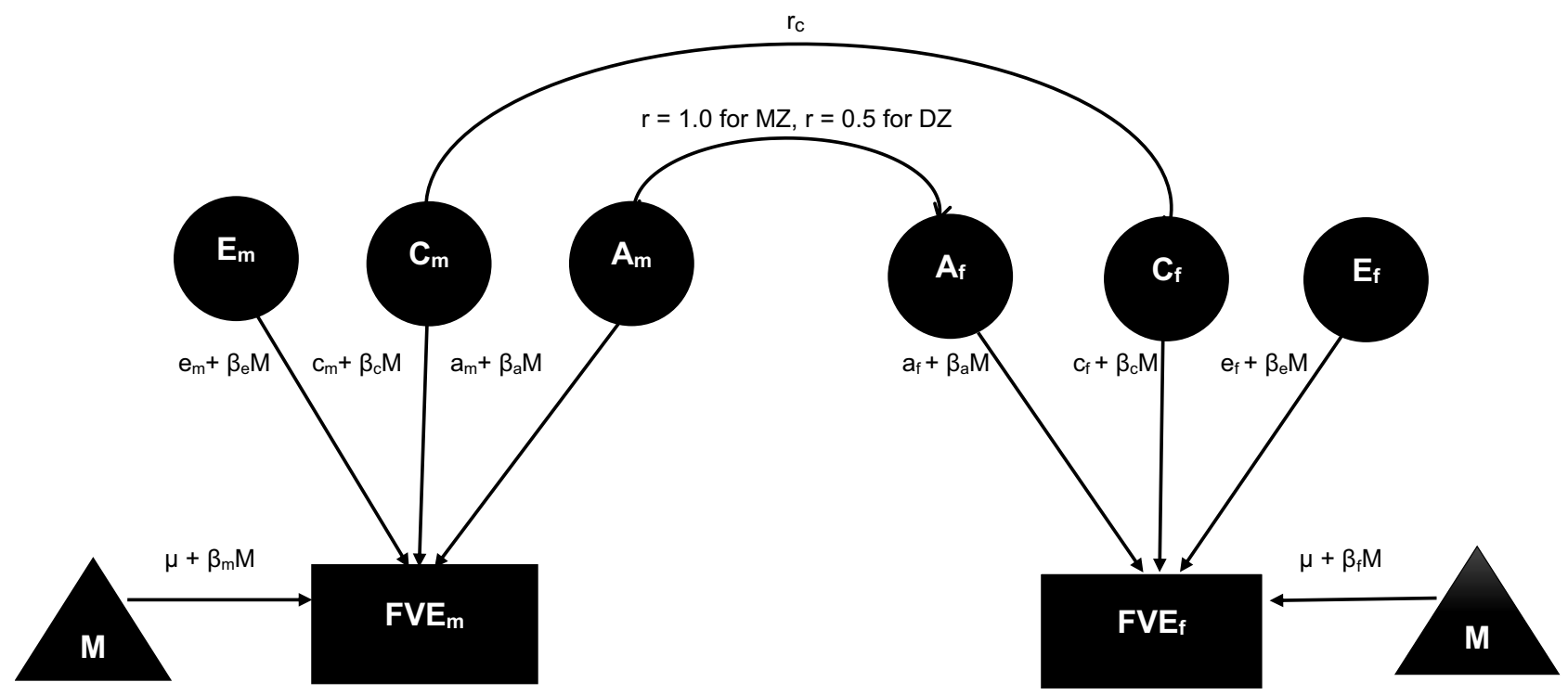

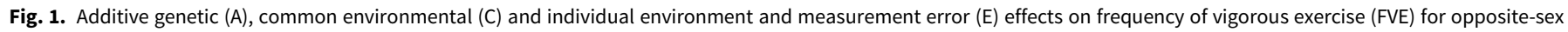

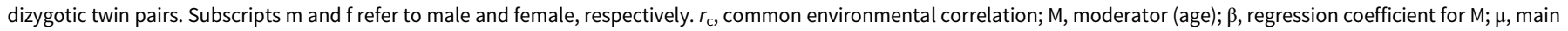
effects of age on FVE.

of zygosity. The moderator variable, age (M) was transformed to a $z$ score separately for males and females, and the main effect of age ( $z$ score) was incorporated on the thresholds for males and females $\left(\mu+\beta_{\mathrm{m}} \mathrm{M}, \mu+\beta_{\mathrm{f}} \mathrm{M}\right)$. In addition, the model allowed the standard paths, A, C and E on the FVE to vary as a function of age ( $z$ score) and sex. Thus, the phenotypic variance $(\mathrm{Vp})$ for each sex in the full age-moderation model can be expressed as $\mathrm{Vp}=\left(\mathrm{a}+\beta_{\mathrm{a}} \mathrm{M}\right)^{2}+\left(\mathrm{c}+\beta_{\mathrm{c}} \mathrm{M}\right)^{2}+\left(\mathrm{e}+\beta_{\mathrm{e}} \mathrm{M}\right)^{2}$. In this equation, $\beta_{\mathrm{a}}, \beta_{\mathrm{c}}$ and $\beta_{\mathrm{e}}$ represent the magnitude of the moderating effects related to $\mathrm{A}, \mathrm{C}$ and $\mathrm{E}$, respectively. Quantitative sex differences were tested by allowing the magnitudes of $\mathrm{A}, \mathrm{C}$ and $\mathrm{E}$ effects to differ between males and females. In addition, the correlation between common environmental effects in OSDZ twin pairs was allowed to be less than 1.0, enabling estimation of qualitative sex difference in common environmental effects (i.e. partly different common environments operate in males and females). To determine the best-fitting, most parsimonious model, various constraints were imposed on the full model, and the resulting nested models were compared with the full model by the log likelihood ratio test (LRT) and Akaike's information criteria (AIC). LRT evaluates the difference in minus two times the log likelihood (-2LL) between the full model and its nested model, which is asymptotically distributed as $\chi^{2}$ with degrees of freedom $(d f)$ equal to the two model's difference in the degrees of freedom. Models with fewer parameters are preferred if they do not cause a significant deterioration of fit. Furthermore, the model with lowest AIC was considered the best-fitting because lower AIC value indicates better balance between parsimony and goodness-of-fit (Akaike, 1987). Statistical software package, $\mathrm{Mx}$ (Neale et al., 2003) was used to conduct twin tetrachoric correlation and model-fitting analysis.

\section{Results}

\section{Descriptive Statistics}

Sex differences in frequency of vigorous exercise. Figure 2 shows sex differences in FVE in the total sample. Pearson chisquare tests showed significant sex differences in the total sample $\left(\chi_{(3)}^{2}=119.7, p<.001\right)$, indicating that males engage in vigorous exercise more frequently than females. Post hoc tests showed that frequency of 'no exercise' was significantly $(p<.05)$ higher in females than in males. In contrast, frequencies of the two highest categories (i.e. 3-4 times per week, 5 times per week or more) were significantly $(p<.05)$ higher in males than in females. Frequencies of the modest exercise category (i.e. 1-2 times per week) did not differ significantly between the two sexes.

The relationship between age and frequency of vigorous exercise. The relationship between age and FVE was modest but significant in both sexes. The correlations were -.12 $(p<.01)$ in males and $-.11(p<.01)$ in females, suggesting that in both sex groups, twins exercise less with increasing age.

Frequency of vigorous exercise by zygosity. A Pearson chisquare test showed no significant difference between $\mathrm{MZ}$ and DZ twins $\left(\chi_{(3)}^{2}=2.3, p<.51\right)$, fulfilling the assumption for twin analysis. Frequencies for MZ and DZ twins were, respectively, $37 \%$ and $34 \%$ for the first response category (i.e. no exercise), $34 \%$ and $31 \%$ for the second response category (i.e. 1-2 times per week), $18 \%$ and $21 \%$ for the third response category (i.e. 3-4 times per week) and $12 \%$ and $14 \%$ for the final category (i.e. 5 times or more per week).

\section{Twin Correlations}

Figure 3 displays tetrachoric twin correlations and their 95\% confidence intervals (CI) for the five zygosity groups. MZM twin correlation $(r=.40,95 \%$ CI $[.22, .55])$ was significant and greater than DZM twin correlation $(r=.12,95 \%$ CI $[-.17, .38])$, suggesting the presence of genetic influences on vigorous exercise in males. In contrast, the MZF correlation $(r=.44,95 \%$ CI $[.32, .54])$ was similar to the DZF correlation $(r=.45,95 \%$ CI $[.27, .60])$, suggesting the presence of common environmental influences. The OSDZ twin correlation $(r=.12 .95 \% \mathrm{CI}[-.09, .31])$ was lower than the DZF correlation but higher than the DZM correlation. These patterns of twin correlations suggested that 


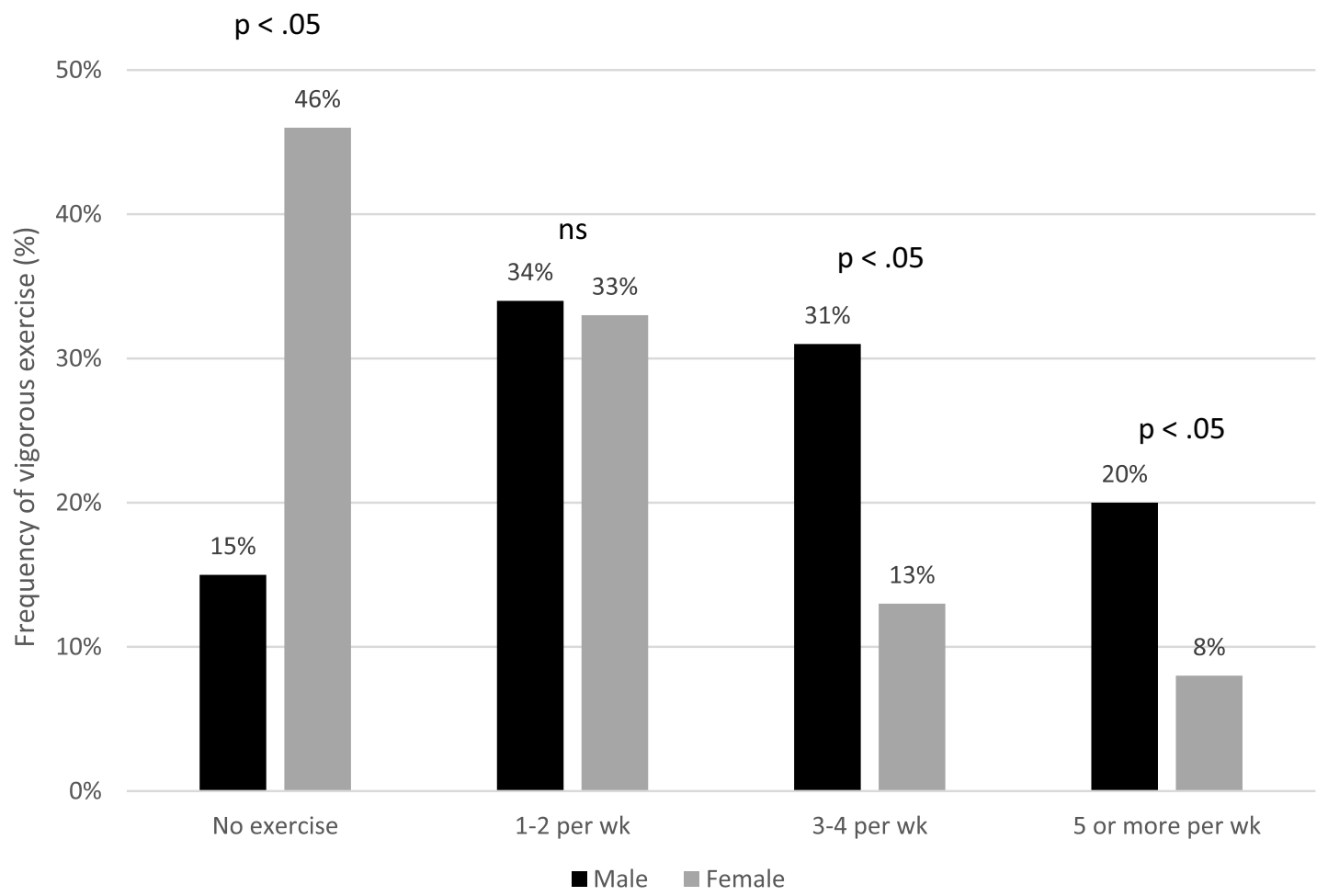

Fig. 2. Frequency of vigorous exercise per week in males (black) and females (gray) in the total sample. ns, not significant.

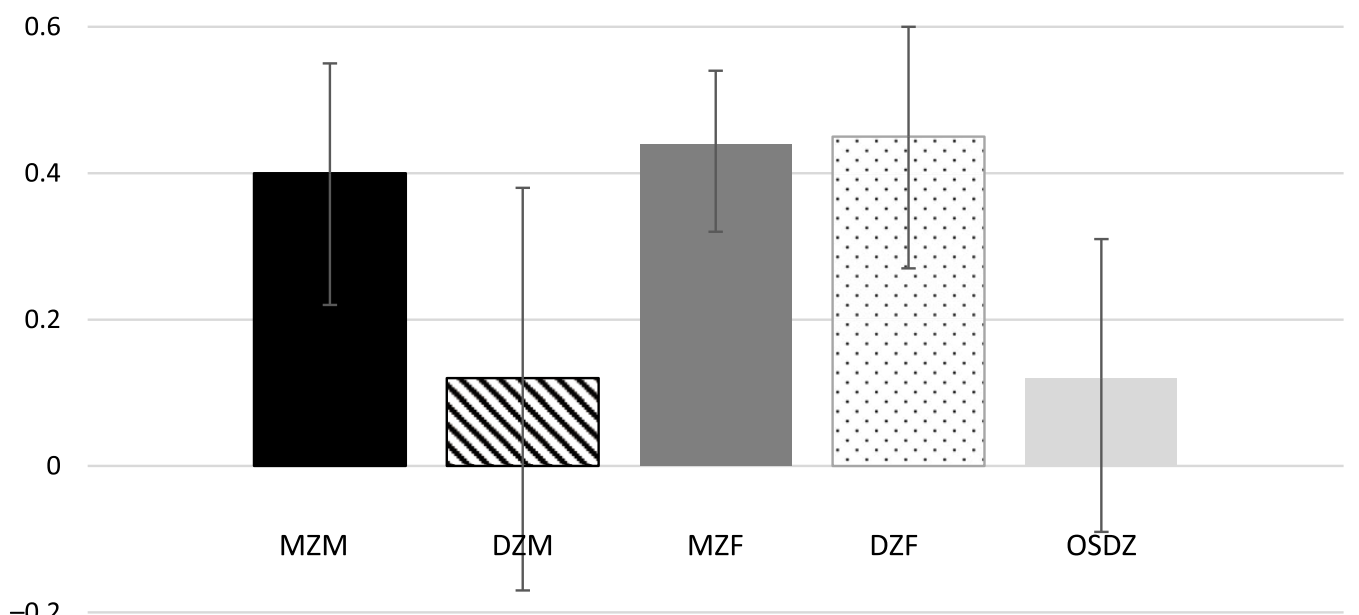

Fig. 3. Tetrachoric correlations ( $95 \% \mathrm{Cl}$ ) for frequency of vigorous exercise per week for MZM (monozygotic male), DZM (dizygotic male), MZF (monozygotic female), DZF (dizygotic female) and OSDZ (opposite-sex dizygotic) twins.

genetic influences were important for individual differences in vigorous exercise in males, whereas common environmental influences were significant in females. MZM and MZF twin correlations were much lower than unity, indicating that individual environmental influences are high in both males and females.

\section{Model Fitting}

Table 1 presents results of model-fitting analysis. When thresholds were equated across the two sexes from the full model, a significant difference in chi-square occurred $\left(\chi_{(3)}^{2}=149.63, p=.00\right)$, confirming sex differences in FVE (model 2). In model 3, qualitative sex difference in common environmental influences was tested. But the resulting chi-square difference was nonsignificant $\left(\chi_{(1)}^{2}=.11, p=.73\right)$. In model 4 , dropping all age moderators from both sexes did not significantly deteriorate model fit $\left(\chi_{(7)}^{2}=10.34\right.$, $p=.17$ ), suggesting that additive genetic, common environmental and individual environmental variances of FVE do not significantly change with age during adolescence and young adulthood. In model 5, additive genetic and common and individual environmental variances were equated across two sexes, which significantly worsened model fit $\left(\chi_{(10)}^{2}=20.93, p=.02\right)$. These results suggested that males and females differed in magnitudes of additive genetic and common and individual environmental 
Table 1. Results of model-fitting analysis for frequency of vigorous exercise per week

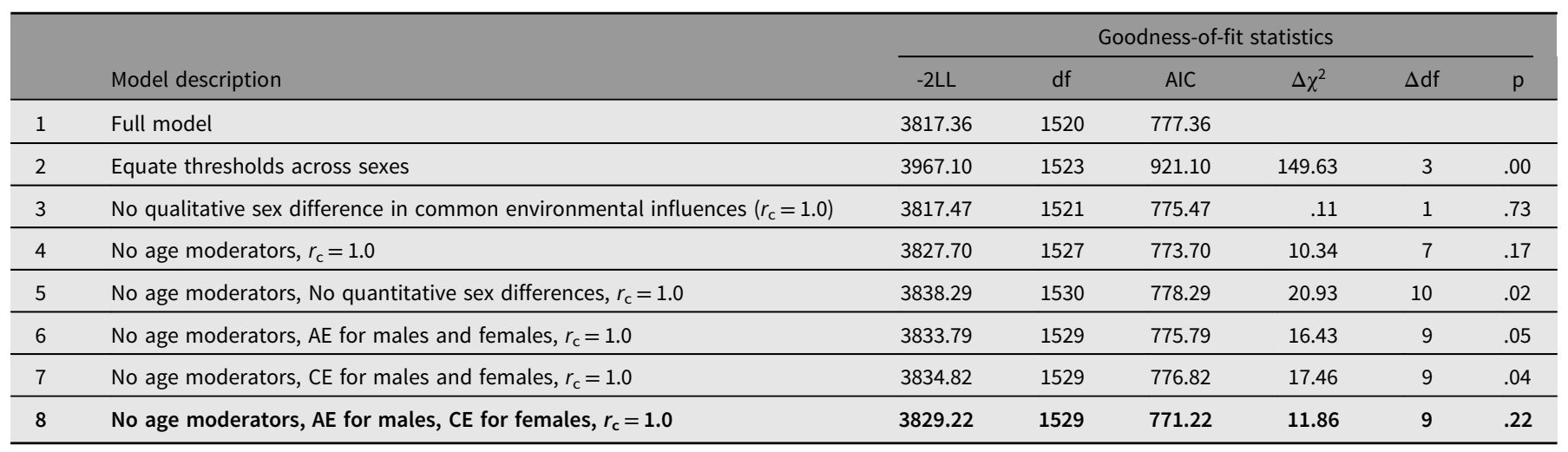

$-2 \mathrm{LL},-2$ log likelihood; A, additive genetic influences; C, common environmental influences; $\mathrm{E}$, individual environmental influences including measurement error. The best-fitting model is indicated in bold type.

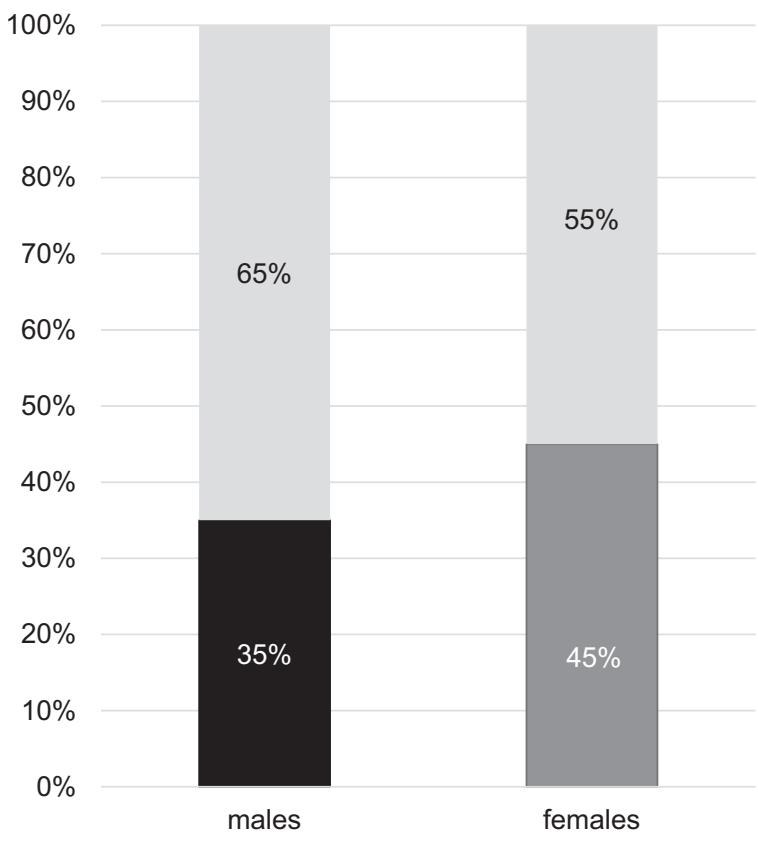

Fig. 4. Additive genetic (A; black), common environmental ( $C$; dark gray) and individual environmental influences including measurement error ( $\mathrm{E}$; light gray) on frequency of vigorous exercise per week in the best-fitting model for males and females. $95 \%$ confidence intervals are in parenthesis.

variances. In model 6, common environmental variances were removed from both sex groups, whereas in model 7 , additive genetic variances were eliminated. In both models, marginally significant chi-square differences occurred $\left(\chi_{(9)}^{2}=16.43, p=.05\right.$ for model $6 ; \chi_{(9)}^{2}=17.46, p=.04$ for model 7). However, a clearly nonsignificant chi-square difference $\left(\chi^{2}{ }_{(9)}=11.86, p=.22\right)$ was found in model 8 , in which common environmental influences were removed from males and additive genetic influences, from females. This suggested preference of model 8 over models 6 or 7. Indeed, among all models tested, model 8 had the lowest AIC value. Thus, model 8 was the best-fitting, most parsimonious according to LRT and AIC. In the best-fitting model (Figure 4), additive genetic and individual environmental influences were, respectively, .35 (95\% CI $[.26, .39])$ and .65 (95\% CI $[.61, .74])$ in males, and common and individual environmental influences were, respectively, .45 (95\% CI $[.35, .53])$ and .55 (95\% CI $[.47, .65])$.

\section{Discussion}

Consistent with prior Western and South Korean singleton samples (e.g. Cho et al., 2014; Sallis et al., 2000), this study showed that the prevalence of vigorous exercise was higher in males than in females. This study further showed that sex difference was driven largely by differences in the extreme groups (i.e. the no exercise group and the two high exercise groups); the modest exercise group showed no significant sex difference. These findings suggest that intervention should focus on no exercisers and intensive exercisers to reduce the gender gap in physical activities.

The present study showed that FVE declined with age in both sexes, which was also in line with many previous studies (e.g. van der Aa et al., 2010). South Korean adolescents are obliged to participate in 1-4 hours of physical education class at school per week, and the hours of compulsory physical education class are lower in higher grades than in lower grades. Thus, the present findings of age-related decline in FVE likely reflected the decrease of compulsory physical education at school with increasing age. In young adults, the transition from student to work life occurs, which may also increasingly constrain the time to engage in vigorous physical exercise.

The finding that genetic influences were higher and common environmental influences were lower in males than in females agreed with results of several previous twin studies based on Western samples (Carlsson et al., 2006; Maia et al., 2002; van der Aa et al., 2010), but contrasted with results from the Wang et al. (2016) study based on Chinese twins. The present study differed from the Wang et al. study in age of sample $(12.42$ -29.42 years vs. 18 years or older) and number of thresholds ( 1 vs. 3 ) in the measure of vigorous physical exercise. To make the measure of this study more comparable to that in the Chinese twin study, the data were reanalyzed using a single threshold model (dichotomy) with a cut-point of FVE of five times or more per week, corresponding to about 150 minutes or more activity per week as in the Chinese twin study. The best-fitting model still showed significant genetic influences in males (49\%) and significant common environmental influences (50\%) in females with the remaining variances attributable to individual environmental influences including measurement error $(51 \%$ in males, $50 \%$ in females) in both sexes. Age differences between the Chinese and 
the present sample might have contributed to the differing outcomes to some degree. However, further exploration of genetic and environmental etiologies of physical activity in Asians is necessary to draw a firm conclusion.

Previous research has suggested that personality traits (e.g. sensation-seeking) (Sallis et al., 2000), self-efficacy (Lee et al., 2018), affective response to exercise (Schutte et al., 2017), physical fitness traits (e.g. muscle strength, muscle endurance and aerobic power) (Bauman et al., 2012) are key factors in maintaining regular physical exercise. Because all of these traits have been shown to be highly heritable (e.g. Polderman et al., 2015), genes influencing them might be involved in the genetic variation for physical exercise in males. To date, genome-wide association studies have found a few loci significantly associated with physical exercise, but effect sizes of the genetic variants found were small (Hara et al., 2018; Klimentidis et al., 2018; de Moor et al., 2009). In the first genomewide association study (GWAS), de Moor et al. (2009) found significant associations of physical exercise with the PAPSS2 gene involved in skeletal development and arthrosis and the SGIP1 gene primarily expressed in the hypothalamus of the brain in Dutch and American samples. Subsequently, Klimentidis et al. (2018) showed that habitual physical activity was significantly associated with higher Alzheimer's risk gene (APOE) and CADM2 gene related to higher body mass index and risk-taking behavior. In a large Japanese sample, Hara et al. (2018) found a novel association between an SNP in the NPSR1-DPY19L1 intergenic region on chromosome 7 and leisure-time exercise behavior.

In females, common environmental influences were more important than genetic influences, explaining about $45 \%$ of variation of FVE in the present sample. Genetic environmental influences may show up later in life in females. Common environmental influences include parental supports and role models, sibling physical activity, family socioeconomic status and neighborhood recreational environment and exercise facilities (e.g. parks and trails, community health center). A review of 108 studies on factors influencing adolescents' physical activity levels suggested that parental support and help were the most consistently associated with adolescents' physical activity levels (Sallis et al., 2000). Parental support and help vary across families, which may constitute common environmental variance in FVE. Although boys tend to receive more parental support in exercise activities than girls (Beets et al., 2010; Gustafson \& Rhodes, 2006), the present findings suggest that parental support and help exert significant influence only on girls. In line with the present study, Sallies et al. (1999) have shown that the effect of parents on sports participation is greater for girls than for boys. In a similar vein, Hanson and Chen (2007) suggest that males are physically more active while interacting with their peers, whereas females are more active in structured activities that are more likely to involve parental influence. In young adults, although parents' direct influence might diminish, the level of a co-twin's physical activity might be important for maintenance of exercise especially for females. Taken together, present findings suggest that family-based interventions might be more useful for increasing exercise in females than in males in adolescents and young adults.

The present study has several limitations that need to be addressed. First, the present study was based on cross-sectional twin data. Decreased physical activity with age might reflect an increase in exercise in younger birth cohorts rather than aging effects. However, a decline in physical activities with age has been confirmed in previous longitudinal studies (Aaltonen et al., 2013; van Mechelen et al., 2000). Second, as in most twin studies, the present study analyzed self-report data. Although regular exercise can be reliably measured by self-report in adolescents and adults (Stubbe et al., 2006), future twin studies using Asian samples might consider replicating the present findings using objective measures, for instance, movement sensors in smartphones. Finally, the generalizability of the present findings is limited because the sample consisted only of South Korean adolescents and young adults.

To conclude, although genetic and common environmental influences on FVE did not vary with age, they differed across males and females in South Korean twins during adolescence and young adulthood. These results suggest that intervention strategies to promote physical activity should be sex-specific.

Acknowledgments. Special thanks are given to twins who participated in the South Korean Twin Registry.

Financial support. This study was supported by the National Research Foundation of Korea grant (NRF-371-2011-1 B00047) and by the 'Development of Health Prediction Technology based on Big Data' (K18092) funded by the Ministry of Science and ICT(MSIT) of Korea given to the Korea Institute of Oriental Medicine (KIOM).

\section{References}

Aaltonen, S., Latvala, A., Jelenkovic, A., Rose, R. J., Kujala, U. M., Kaprio, J., \& Aaltonen, S. (2020). Physical activity and academic performance: Genetic and environmental associations. Medicine and Science in Sports and Exercise, $52,381-390$.

Aaltonen, S., Ortega-Alonso, A., Kujala, U. M., \& Kaprio, J. (2013). Genetic and environmental influences on longitudinal changes in leisure-time physical activity from adolescence to young adulthood. Twin Research and Human Genetics, 16, 535-543.

Akaike, H. (1987). Factor analysis and AIC. Psychometrika, 52, 317-332.

Aune, D., Norat, T., Leitzmann, M., Tonstad, S., \& Vatten, L. J. (2015). Physical activity and the risk of type 2 diabetes: A systematic review and dose-response meta-analysis. European Journal of Epidemiology, 30, 529-542.

Bartels, M., de Moor, M. H., van der Aa, N., Boomsma, D. I., \& de Geus, E. J. (2012). Regular exercise, subjective wellbeing, and internalizing problems in adolescence: Causality or genetic pleiotropy? Frontier Genetics, 3, 4.

Bauman, A. E., Reis, R. S., Sallis, J. F., Wells, J. C, Loos, R. J. F., \& Martin, B. W. (2012). Lancet Physical Activity Series Working Group. Correlates of physical activity: Why are some people physically active and others not? Lancet, 380, 258-271.

Beets, M. W., Cardinal, B. J., \& Alderman, B. L. (2010). Parental social support and the physical activity-related behaviors of youth: A review. Health Education \& Behavior, 37(5), 621-644.

Brown, J. C., Winters-Stone, K., Lee, A., \& Schmitz, K. H. (2012). Cancer, physical activity, and exercise. Comprehensive Physiology, 2, 2775-2809.

Carlsson, S., Andersson, T., Lichtenstein, P., \& Michaelsson, K. (2006). Genetic effects on physical activity: Results from the Swedish Twin Registry. Medicine and Science in Sports and Exercise, 38, 1396-1401.

Cho, K. O., Lee, S., \& Kim, Y. S. (2014). Physical activity and sedentary behavior are independently associated with weight in Korean adolescents. Journal of Lifestyle Medicine, 4, 47-54.

de Geus, E. J., Bartels, M., Kaprio, J., Lightfoot, J. T., \& Thomis, M. (2014). Genetics of regular exercise and sedentary behaviors. Twin Research and Human Genetics, 17, 262-271.

de Moor, M. H., Liu, Y. J., Boomsma, D. I., Li, J., Hamilton, J. J., Hottenga, J. J., Levy, S., Liu, X. G., Pei, Y. F., \& Posthuma, D. (2009). Genome-wide association study of exercise behavior in Dutch and American adults. Medicine and Science in Sports and Exercise, 41, 1887-1895.

de Moor, M. H. M., Willemsen, G., Rebollo-Mesa, I., Stubbe, J. H., de Geus, E. J. C., \& Boomsma, D. I. (2011). Exercise participation in 
adolescents and their parents: evidence for genetic and generation specific environmental effects. Behavior Genetics, 41, 211-222.

Grasdalsmoen, M., Eriksen, H. R., Lønning, K. J., \& Sivertsen, B. (2020). Physical exercise, mental health problems, and suicide attempts in university students. BMC Psychiatry, 20, 175.

Gustafson, S. L., \& Rhodes, R. E. (2006). Parental correlates of physical activity in children and early adolescents. Sports Medicine, 36, 79-97.

Guthold, R., Stevens, G. A., Riley, L. M., \& Bull, F. C. (2018). Worldwide trends in insufficient physical activity from 2001 to 2016: A pooled analysis of 358 population-based surveys with 19 million participants. Lancet Global Health, 6, e1077-e1086.

Hanson, M. D., \& Chen, E. (2007). Socioeconomic status and health behaviors in adolescence: a review of the literature. Journal of Behavioral Medicine, 30, 263-285.

Hara, M., Hachiya, T., Sutoh, Y., Matsuo, K., Nishida, Y., Shimanoe, C., Tanaka, K., Shimizu, A., Ohnaka, K., Kawaguchi, T., Oze, I., Matsuda, F., Ito, H., Kawai, S., Hishida, A., Okada, R., Sasakabe, T., Hirata, A., Ibusuki, R., ... Wakai, K. (2018). Genome-wide association study of leisure-time exercise behavior in Japanese adults. Medicine and Science in Sports and Exercise, 50, 2433-2441.

Hills, A. P., Andersen, L. B., \& Byrne, N. M. (2011). Physical activity and obesity in children. British Journal of Sports Medicine, 45, 866-870.

Hur, Y-M., Kang, M. C., Jeong, H-U., Kang, I. C., \& Kim, J. W. (2019). The South Korean Twin Registry. Twin Research and Human Genetics, 22, 606-608.

Hur, Y.-M., \& Kwon, J. S. (2005). Changes in twinning rates in South Korea: 1981-2002. Twin Research and Human Genetics, 8, 76-79.

Hur, Y.-M., \& Song, T. B. (2009). A recent rise in twin birth rates and demographic changes in mothers of twins in South Korea: 2003-2007. Twin Research and Human Genetics, 12, 118-122.

Iso-Markku, P., Waller, K., Vuoksimaa, E., Vähä-Ypyä, H., Lindgren, N., Heikkilä, K., Sievänen, H., Rinne, J., Kaprio, J., \& Kujala, U. M. (2018). Objectively measured physical activity profile and cognition in Finnish elderly twins. Alzheimers \& Dementia, 4, 263-271.

Klimentidis, Y. C., Raichlen, D. A., Bea, J., Garcia, D. O., Wineinger, N. E., Mandarino, L. J., Alexander, G. E., Chen, Z., \& Going, S. B. (2018). Genome-wide association study of habitual physical activity in over 277,000 UK Biobank participants identifies multiple variants including CADM2 and APOE. International Journal of Obesity (Lond), 42, 1161-1176.

Lee, C. G., Park, S., Lee, S. H., Kim, H., \& Park, J.-W. (2018). Social cognitive theory and physical activity among Korean male high-school students. American Journal of Men's Health, 12, 973-980.

Maia, J. A. R., Thomis, M., \& Beunen, G. (2002). Genetic factors in physical activity levels: a twin study. American Journal of Preventive Medicine, 23, $87-91$.
Neale, M. C., Boker, S. M., Xie, G., \& Maes, H. H. (2003). Mx: Statistical Modeling (5th ed.). Virginia Commonwealth University, Department of Psychiatry.

Ooki, S., Yamada, K., \& Asaka, A. (1993). Zygosity diagnosis of twins by questionnaire for twins' mothers. Acta Geneticae Medicae et Gemellologiae (Roma), 42, 17-22.

Polderman, T. J., Benyamin, B., de Leeuw, C. A., Sullivan, P. F., van Bochoven, A., Visscher, P. M., \& Posthuma, D. (2015). Meta-analysis of the heritability of human traits based on fifty years of twin studies. Nature Genetics, 47, 702-709.

Sallis, J. F., Prochaska, J. J., \& Taylor, W. C. (2000). A review of correlates of physical activity of children and adolescents. Medicine and Science in Sports and Exercise, 32, 963-975.

Sallies, J. K., Alcaraz, J. E., Mckenzie, T. L., \& Hovell, M. F. (1999). Predictors of change in children's physical activity over 20 months: Variations by gender and level of adiposity. American Journal of Preventive Medicine, $16,222-229$

Schutte, N. M., Nederend, I., Hudziak, J. J., Bartels, M., \& de Geus, E. J. C. (2017). Heritability of the affective response to exercise and its correlation to exercise behavior. Psychology of Sport and Exercise, 31, 139-148.

Stubbe, J. H., Boomsma, D. I., Vink, J. M., Cornes, B. K., Martin, N. G., Skytthe, A., Kyvik, K. O., Rose, R. J., Kujala, U. M., Kaprio, J., Harris, J. R., Pedersen, N. L., Hunkin, J., Spector, T. D., \& de Geus, E. J. C. (2006). Genetic influences on exercise participation in 37.051 twin pairs from seven countries. PLoS ONE, 1, e22.

van der Aa, N., de Geus, E. J. C., van Beijsterveldt, C. E. M., Boomsma, D. I., \& Bartels, M. (2010). Genetic influences on individual differences in exercise behavior during adolescence. International Journal of Pediatrics, 138345. doi: 10.1155/2010/138345.

van Mechelen, W., Twisk, J. W., Post, G. B., Snel, J., \& Kemper, H. C. (2000). Physical activity of young people: The Amsterdam Longitudinal Growth and Health Study. Medicine and Science in Sports and Exercise, $32,1610-1616$.

Vink, J. M., Boomsma, D. I., Medland, S. E., de Moor, M. H. M., Stubbe, J. H., Cornes, B. K., Martin, N. G., Skytthea, A., Kyvik, K. O., Rose, R. J., Kujala, U. M., Kaprio, J., Harris, J. R., Pedersen, N. L., Cherkas, L., Spector, T. D., \& de Geus, E. J. C. (2011). Variance components models for physical activity with age as modifier: A comparative twin study in seven countries. Twin Research and Human Genetics, 14, 25-34.

Wang, B., Gao, W., Lv, J., Yu, C., Wang, S., Pang, Z., Cong, L., Dong, Z., Wu, F., Wang, H., Wu, X., Jiang, G., Wang, X., Wang, B., Cao, W., \& Li, L. (2016). Physical activity attenuates genetic effects on BMI: Results from a study of Chinese adult twins. Obesity, 24, 750-756.

Wannamethee, S. G., \& Shaper, A. G. (2001). Physical activity in the prevention of cardiovascular disease: An epidemiological perspective. Sports Medicine, 31, 101-114. 